\title{
Cloud Computing in Science and Engineering and the "SciShop.ru" Computer Simulation Center
}

\author{
Gennady A. Tarnavsky \\ Institute of Computational Mathematics and Mathematical \\ Geophysics, \\ Siberian Branch of the Russian Academy of Sciences \\ Novosibirsk, Russia \\ Gennady.Tarnavsky@gmail.com
}

\author{
Evgenii V. Vorozhtsov \\ Khristianovich Institute of Theoretical and Applied \\ Mechanics, \\ Siberian Branch of the Russian Academy of Sciences \\ Novosibirsk, Russia \\ vorozh@itam.nsc.ru
}

\begin{abstract}
Various aspects of cloud computing applications for scientific research, applied design, and remote education are described in this paper. An analysis of the different aspects is performed based on the experience from the "SciShop.ru" Computer Simulation Center. This analysis shows that cloud computing technology has wide prospects in scientific research applications, applied developments and also remote education of specialists, postgraduates, and students.
\end{abstract}

Keywords-cloud computing; information technologies; internet; computer simulation

\section{INTRODUCTION}

One can identify Cloud Computing as a unique direction among the various developments of advanced information technologies. It should be noted that the cloud is one of the internet symbols. The purpose of the present work is to give a general review of Cloud Computing and to illustrate an example based on the internet cloud portal, "SciShop.ru" Computer Simulation Center.

\section{A. The Ideology of Cloud Computing}

Cloud Computing is a rapidly developing and promising direction of contemporary informatics. The ideology of cloud computations lies in transferring, to a considerable extent, the arrangement for computations and the processing of data from personal computers to Worldwide Web servers. The concept of Cloud Computing is based on the internet being capable of meeting the demands of users as they are generated and also processing data within wide ranges of users' requests. For example, the Google Apps system provides applications for business in the online regime, the access to which occurs with the aid of internet browsers, and all software and data are stored in the Google servers whereas the Google Chrome operation system (OS) is based entirely on cloud computing. In addition, the Microsoft Corporation has developed its new version of the OS Windows Azure whose segments are based nearly entirely on cloud technologies. In particular, MS Office is stored in MS servers, with a free (licensed) access provided to clients on their request. This frees the users from the need to install the system in their own computers and to support its functioning in the future.

The resource demands for PCs and even for workstations for collective use, decrease substantially when using cloud computing. The Cloud Computing direction is exceptionally promising for the development of remote education in different branches of knowledge as well as for computer modeling in the remote access regime. Therefore, one should expect in the near future a massive appearance of cloud Web resources.

\section{B. Technologies of Cloud Computing}

Cloud Computing contains a specialized spectrum of processing and transferring data technologies with computer resources being provided to the user as internet services. The user has access to data, which are permanently stored in Web servers, only as a client during the internet sessions. The information (and process results) can be stored on personal computers, notebooks, netbooks, smartphones, etc. Until now, one can identify several basic technologies (models) of Cloud Computing:

- Infrastructure as a Service (IaaS).

- Platform as a Service (PaaS).

- Data as a Service (DaaS).

- Software as a Service (SaaS).

- Workplace as a Service (WaaS).

- All as a Service (AaaS).

Within the framework of the IaaS, PaaS, DaaS, SaaS, and WaaS models the customers pay for the software lease (the AaaS model), that is its use via the Web interface, rather than for the possession of the software itself. Thus, in contrast to the classical scheme of licensed software purchase, the customer does not need to invest large funds for the acquisition of the product and for a computer platform suitable for its installation and for its future efficient operation. The customer has only relatively low periodic expenses in the form of user charges which can always be interrupted (and renewed) based on the need for the software. 


\section{Cloud Computing in internet}

The process of the transfer of computational complexes conventionally involved the purchase of computer codes (in some cases also the program texts), from the developers, by the future user and their installation on his computer. For a faultfree and smooth operation of the program, the user should have a similar operation environment, including the systems for the visualization of digital data. The stage of program installation can be very complex and often causes many difficulties whose surmounting may require considerable and non-productive expenses of intellectual efforts and time. The Cloud form of using the computational complexes solves all problems related to the installation of the acquired program product. It should be noted that the right of use of program complexes rather than their sale is kept in mind here. The electronic forms of trade in the internet, including the trade of computer programs, are already fairly well developed.

A special Computer Simulation Center site named "SciShop.ru" is currently being developed. The program complexes are hosted in this Center together with all of their attributes: the processing system for the preparation of tasks, the processing system for the execution of tasks (the execution of computational operations) and the postprocessing system for the output of the obtained information in digital and graphical forms. The user has only to formulate its own computational task by inputting the necessary numerical data and start its computation. The problem solution will be provided for the user upon termination of computations. User's comfort during their stay at the Center is ensured via client support. In economic terms, such a method of operation of program complexes means their leasing from the resource developers rather than their purchase.

\section{SITES PROVIDING USERS A DIRECT COMPUTER SIMULATION IN INTERNET REMOTE ACCESS REGIME}

In the near future, one should expect mass appearance of internet sites at which the simulation of subject-oriented large scientific and applied problems related to numerical integration of complex nonlinear equations systems will be carried out. The "SciShop.ru" Computer Simulation Center [1, 2, 3] is such an internet site, providing (at present) solutions for problems of high-speed aerodynamics $[4,5,6,7]$, computational astrophysics [8], and the design of nanostructured semiconductor materials $[9,10]$

The direct numerical computations of problems formulated and started by the visitor of the site are carried out at the center, without an intermediate procedure of the purchase of computational complexes and installation on the visitor's computer. It should be emphasized that all interfaces have been debugged and "tuned" properly and no problems regarding the operation of acquired programs arise when placing the processing systems. The functioning of complexes is troublefree within the indicated ranges of the variation of parameters (note that one can ask on the site forum at any time any question and get an explanation). One more advantage of this approach is that the user is freed from the necessity of buying the often costly hardware, which is necessary for the execution of needed computations [11]. The user "leases" the hardware from the developers of the site only for the time needed for the solution of his problem.

Summarizing the above brief survey one can state the following: the Cloud Computing concept is a very promising direction of infocommunication technology and constantly gain a more and more widespread application range. The introduction of the cloud concept in science and engineering is yet delayed, but one may predict a mass appearance of cloud portals of computer simulation in the future, since Cloud Computing provides appealing advantages regarding the "consumption" of program products compared to conventional commercial sales. It should be noted however that, besides technological problems, special problems of copyright and intellectual property may arise [12].

\section{III. "SCISHOP.RU" COMPUTER SIMULATION CENTER}

The "SchiShop.ru" Computer Simulation Center is an internet cloud portal (Figures 1 and 2). The goal of the development of the Computer Simulation Center (http://www.SciShop.ru) is the development and improvement of new techniques for scientific knowledge dissemination, based on advanced infocommunication technologies for the process, storage, transfer and protection of information.

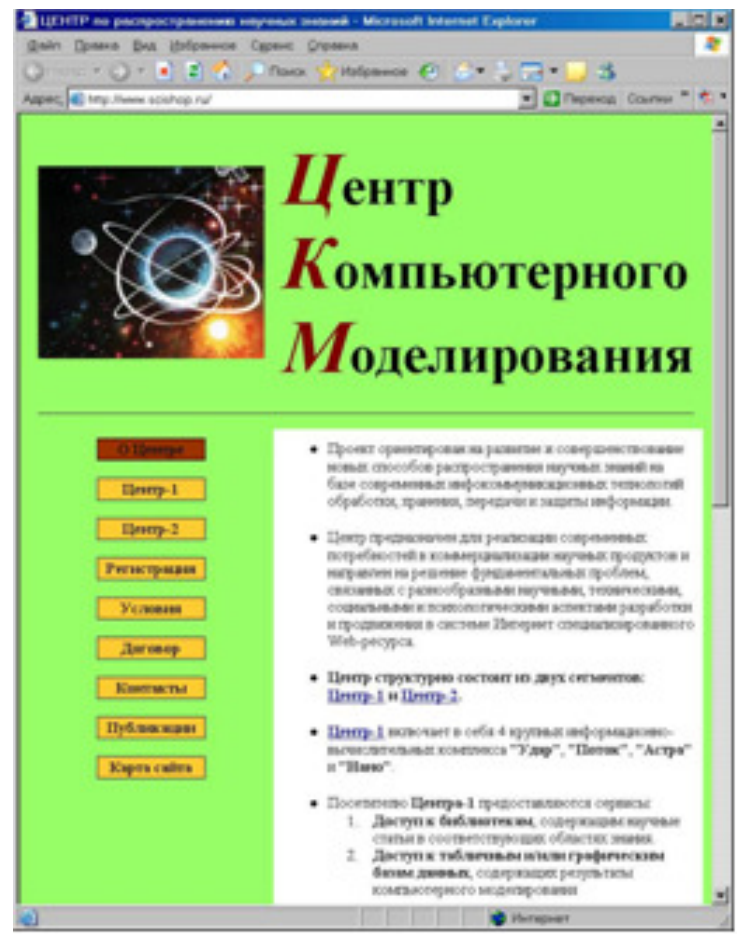

Fig. 1. Main page of the internet center for computer simulation (a fragment).

- The center is designed for the realization of advanced needs in the commercialization of scientific products. It aims to the solution of fundamental problems related to various scientific, technological, social, and psychological aspects 
of the development and promotion of specialized Web resources.

- The center structurally consists of two segments: Center-1 and Center-2.

- The center includes four big information-computation complexes (ICCs) "Impact", "Flow", "Astra", and "Nano", as shown in Figures 3-6.

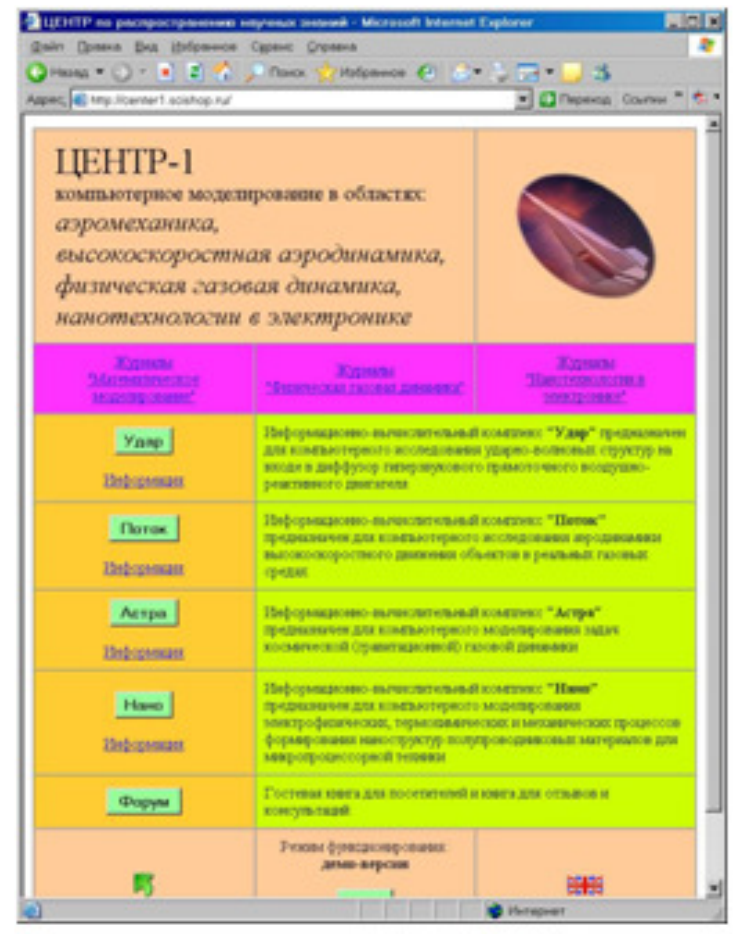

Fig. 2. Main page of the section "Center-1".

\section{A. Services Provided by Center-1}

- The following services are provided for the visitor of Center-1:

1. access to the libraries containing scientific papers on declared themes.

2. access to tabular and/or graphical databases containing the results of computer simulation of some spectrum of computer tasks.

3. access to the processing systems enabling the client to organize and conduct the computer simulation of problems which are of interest to him.

4. access to sites of leading Russian and foreign journals in corresponding branches of knowledge.

5. access to "Forum"' for consultations and discussion of problems.

- In the non-commercial regime, the visitor has access to the demo versions of Center-1 systems. For a full-scale access, the visitor has to register and transfer payment.
- The Center's system for receiving payment accepts payments from any electronic payment systems (WebMoney, Yandex.Money, E-gold, etc.) included in the "Robokassa" consortium. A system for receiving payments using credit bank cards and mobile telephony SMS has also been developed and is currently being verified.

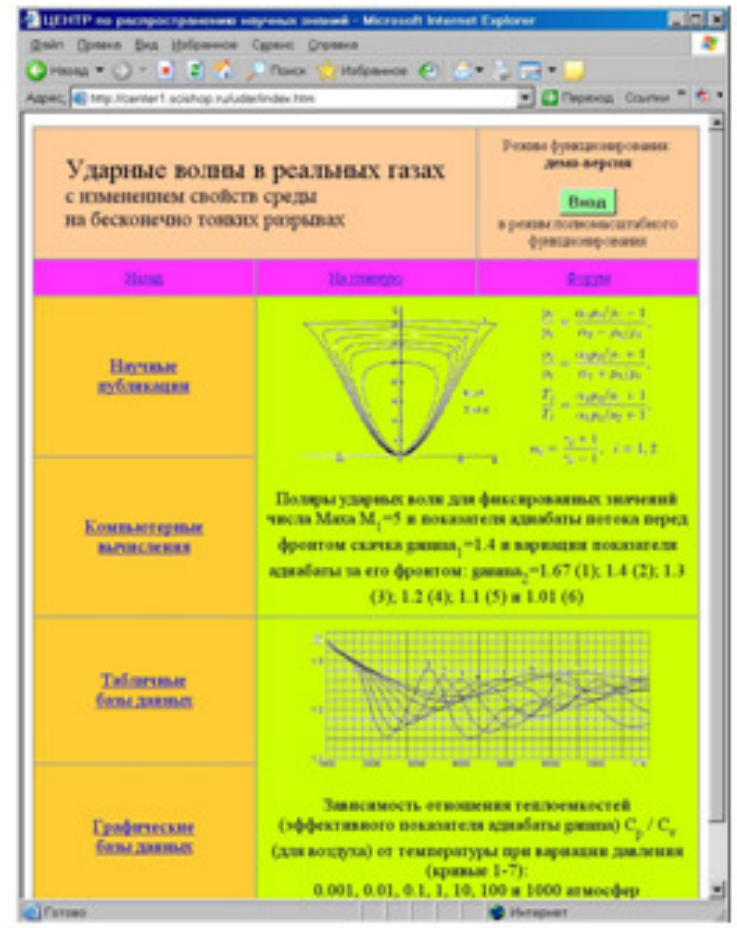

Fig. 3. Base page of the section of the ICC "Impact" "High-velocity internal aerodynamics: computation of shockwave structures in a scramjet diffuser".

\section{B. Services Provided by Center-2}

- Center-2 is intended for storing program developments in various branches of knowledge, without theme limitations. The content of this section can be provided by specialists having the copyright for these information resources.

- All specialists in the area of computer simulation in any knowledge branches, that wish to promote their work on commercial basis, are invited for cooperation with Center2. Specialists wishing to place their scientific products at Center-2 have to study the conditions, get in touch with the administration of the Center and sign an agreement.

One can learn more about all aspects of the Center functions in the special section "Publications" using the corresponding hyperlink from the site main page. All cloud technologies IaaS, PaaS, DaaS, SaaS, WaaS, and AaaS are used at the "SciShop.ru" portal in its different segments. 


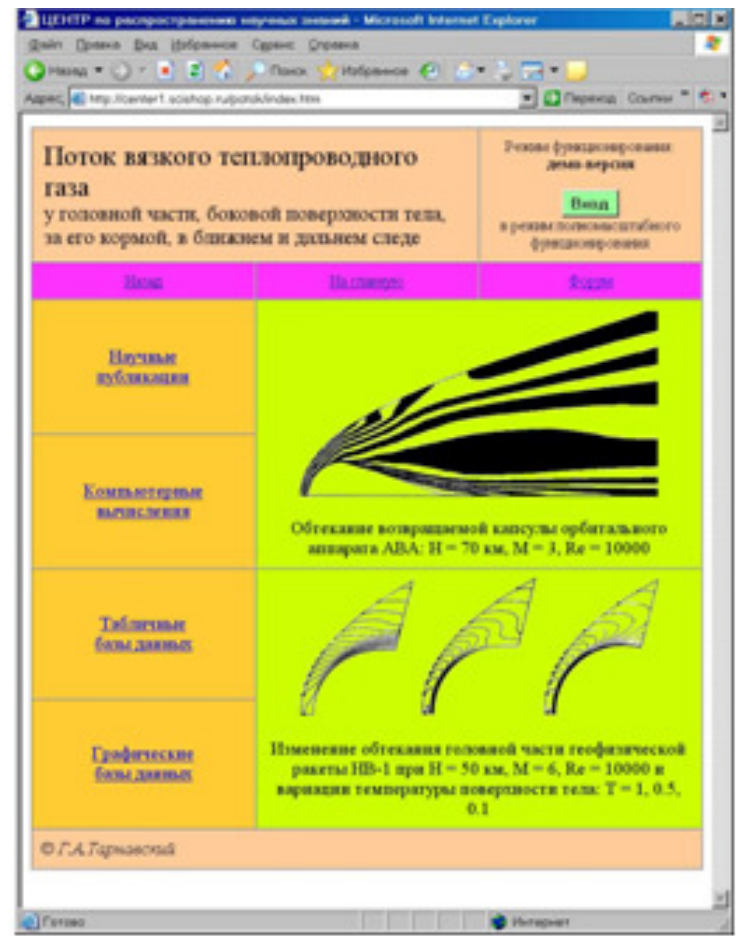

Fig. 4. Base page of the section of the ICC "Flow" "High-velocity external aerodynamics: the computation of the flow around the objects in the atmosphere of Earth and Mars".

\section{C. "Infrastructure as a Service" (IaaS) Technology}

The computer simulation center provides internet infrastructure and portal infrastructure:

- apparatus means (servers, the systems for storing the data, client systems, net facilities).

- operation systems and the system software (the means of virtualization, automation, and control).

- multi-functional segments of the portal content (the switches of regimes, gateways, etc.).

\section{D. "Software as a Service" (SaaS) Technology}

The information-computational complexes provide the client with the following characteristics:

- complexes are used in the remote access regime.

- multi-communication (several clients may use the same complex simultaneously).

- support of complex systems is included in the payment.

- modernization of complexes occurs smoothly and invisibly for clients.

\section{E. "Data as a Service" (DaaS) Technology}

The Center provides to the client the library, tabular, and graphical databases, which contain:
- theoretical materials and the texts of published papers.

- digital results of executed computations of scientific and applied problems and their visualization.

The client is provided with the necessary control of data flow and information protection concerning the transfer of data via the net.

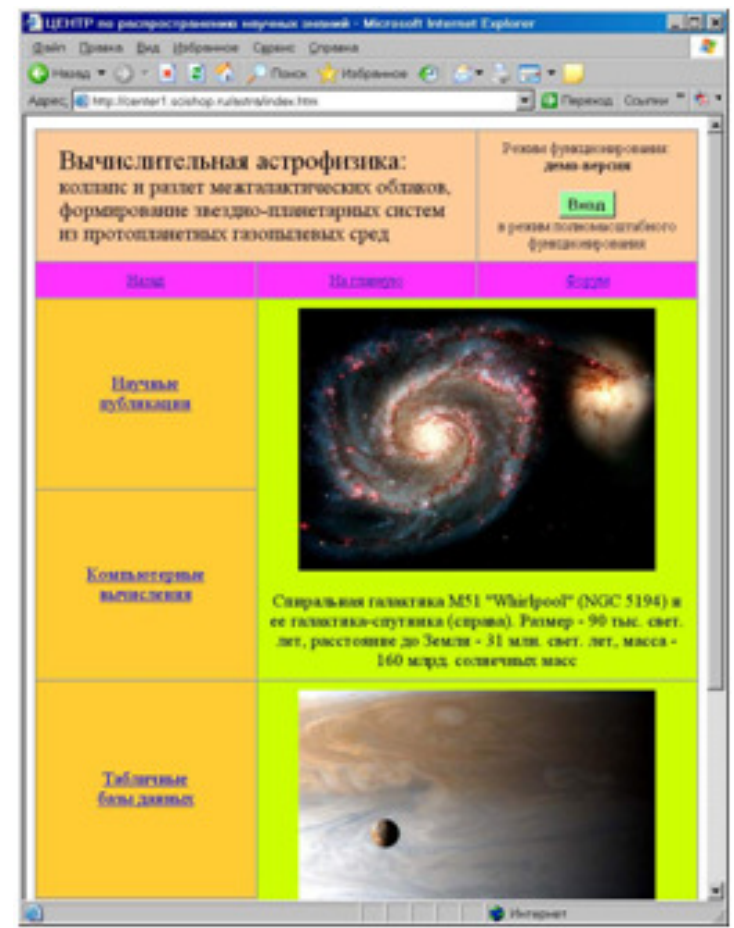

Fig. 5. Base page of the section of the ICC "Astra" "Computational astrophysics: simulation of the dynamics of processes in the inter-galactic gas and protoplanet clouds".

\section{F. "Workplace as a Service" (WaaS) Technology}

The center provides maximum comfort for scientific research to clients and reduces the costs for the operation of the clients' own workstations, by "replacing" them with a virtual environment of centralized infrastructure (employing the supercomputer of the Siberian Branch of the Russian Academy of Sciences).

\section{G. "All as a Service" (AaaS) Technology}

The program complexes of the Center may be used both free of charge (demo versions) and on commercial basis:

- access to full-scale functioning of the Center is granted after the visitor's registration in the specialized "book of record" and after the visitor transfers the user charge via the electronic payment systems.

- the specialized "Robokassa" bank system is used for payment transfers, which ensures the use of more than 20 electronic payment systems (WebMoney, Yandex.Money, Internet.Money, etc.). 
- a system has been introduced, enabling the payments with the use of mobile telephony SMS. Payment transfer safety, routing transparency, the necessary messages for the client and the currency conversion in different electronic payment systems, is ensured by the special structures of the "Robokassa" system and has been checked during the trial operation of the Computer Simulation Center.

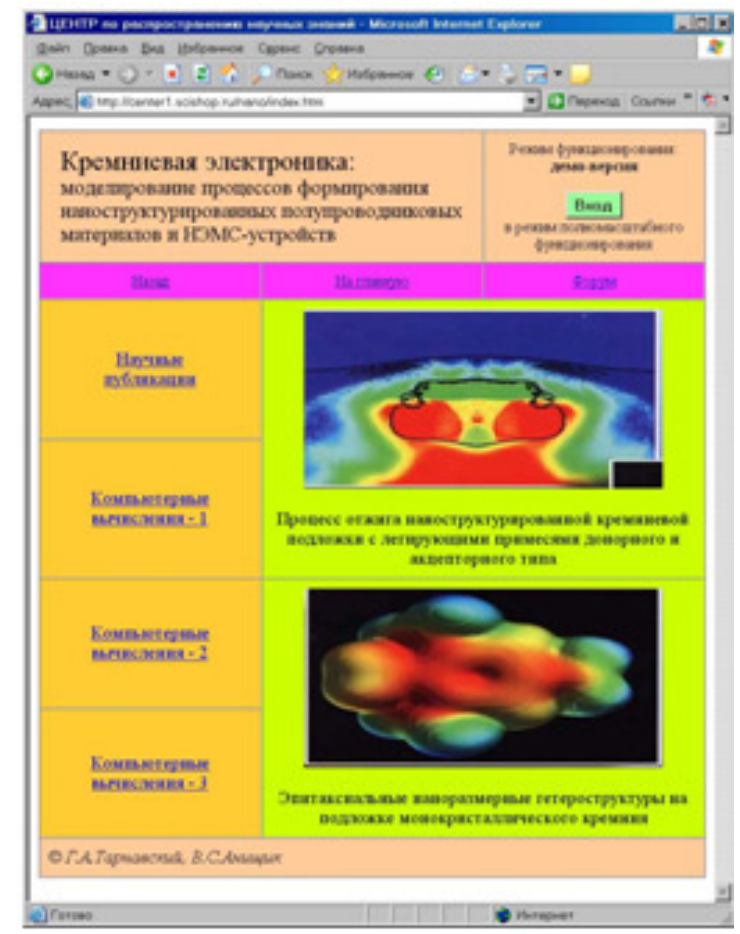

Fig. 6. Base page of the ICC "Nano" "Microelectronics: computer support of the design of nano-structured semiconductor materials".

\section{H. Arrangement of Computations and Information Flows on the Portal. "Data File Cruise" DFC Technology}

The comfort of the client's stay at the internet center and a convenient use of its segments (the bibliographic section, databases and especially the processing systems), are the most important attributes of any program complex. The systems for the preprocessing preparation of tasks (the input of parameters and the start of processing systems) are organized in a clear, convenient, and simple form, which does not cause any ambiguous interpretation and difficulties even for users with small experience. Operations are performed by the user in the remote access regime at the Computer Simulation Center via the Worldwide web rather than in the user's own computer during the installation of the complex. This gives the visitor of the Center the opportunity to study the computational complex in the real time regime, organize the solution of the task of interest, and obtain the result of computer studies.

The computational complex transfer usually involves the acquisition of licenses, documentation, and computer codes. After that, the user installs the purchased product on his own computer. This may cause difficulties in general, due to various reasons, ranging from the use of different versions of operation systems to the peculiarities of the seller's and buyer's supporting systems. Such problems become obsolete with the placing of the processing systems at the Center.

Significantly enhanced Cloud Computing methodology is incorporated at the creation of the Web resource "SciShop.ru". In general, Cloud Computing methodology usually assumes that the information flow moves only in two directions: from the client's browser to the internet server and back. One can call the developed new organization of information flows in "SciShop.ru", the DFC (Data Files Cruise) technology. This technology has a much larger number of motion directions of information flows: from the client's browser to the "SciShop.ru" internet server to the entrance in the supercomputing center (IP1 address) to the exit from the supercomputing center (IP2 address) to the "SciShop.ru" internet server and back to the client's browser. The client can take the obtained solution file either directly at the portal "SciShop.ru" or at the indicated IP2 address. It is planned to implement subsequently a third variant: the transfer of the solution at an address indicated by the client.

The DFC technology is a big advantage for the Computer Simulation Center because the client, possibly a scientific researcher or applied designer, may not be familiar with system programming. This way the user can focus on the physical or technological meaning of his problem, without unjustified and excessive time expenses and intellectual effort for the formation of the computational task. To avoid any misunderstandings it should be noted that the procedure of using the supercomputer in the remote access regime is sufficiently well known, however, quite a different thing is meant here: the creation of the files for the access to the supercomputer, the execution of computations, and solution transfer at the indicated address is done by the systems of the "SciShop.ru" automatically, without the client's participation. It is possible subsequently to also use other supercomputing centers on an alternative and/or combined basis.

\section{Positive AND NEGATIVE ASPECTS OF CLOUD COMPUTING}

Cloud computing application gives rise to a number of complex problems, with several positive and negative arguments. The following positive and negative factors of Cloud Computing can be identified:

\section{- Positive factors for developers:}

1. efficient struggle against the non-licensed use of programs since the product itself does not reach the customer.

2. relative ease of detection and suppression of unauthorized access of several users under the same login.

3. substantial reduction of costs for the development and introduction of technical and consulting support for each customer. 
- Positive factors for users:

1. no need for software installation in the workplaces of the users since access to the software is performed via a plain browser.

2. drastic reduction of the expenses for the unwrapping of the system in an institution.

3. reduction of costs for technical support and renewal of unwrapped systems, including the case of their total absence.

4. quickness of introduction, due to the absence of time expenses for system unwrapping.

5. clarity and predictability of payments.

6. possibility of obtaining a higher level of software servicing.

- Negative factors for the developers:

1. Cloud Computing is by no means applicable to all functional problems.

2. since the main economy of the provider's resources is reached at the expense of the scale, the Cloud Computing approach may prove inefficient for a small number of clients.

3. the Cloud Computing approach is inefficient if it is necessary to perform an in-depth individual adaption to each customer.

- Negative factors for customers:

1. attachment to a single developer and its hosting site.

2. instability of the provider's work may lead to impossibility of long-term planning and deadline wrecking.

3. undesirability of using the cloud computing for projects of strict confidentiality because of a high risk of information leaking from the service provider and the impossibility of controlling the process.

4. difficulty of improving the quality of the services in the current operation regime.

5. need for a permanent internet connection with a fairly high speed of data transfer.

An important issue is the confidentiality problem. The scientific and technological process in any country and, naturally, in Russia has certain "niches" of closed themes where information leaking is extremely undesirable. The outside skilled specialist can easily determine by a number of signs the direction of the research theme. This may obliterate the application of Cloud Computing for the given objectives in principle despite the wide spectrum of positive factors.

Regarding the developer, one of the main shortcomings of Cloud Computing is the high cost of entering the market. To provide a competitive price to the client the developer needs the "scale effect", that is a large number of potential clients.
This way small projects with a convenient very low payment, may have large profit rates depending on the number of clients.

\section{CONCLUSIONS}

In the present paper, we have briefly investigated the ideology, technologies, and problems regarding the Cloud Computing concept, considering the example of the "SciShop.ru" Computer Simulation Center, a pioneer of a new form of scientific knowledge dissemination. This Center is designed for direct use of program complexes of mathematical modeling of processes in various scientific areas, providing the possibility of a direct execution of computations in the internet remote access regime. Such a form has wide prospects of application in scientific research as well as applied developments for the remote education of specialists, postgraduates, and students.

\section{REFERENCES}

[1] G. A. Tarnavsky, A. V. Aliev, V. S. Anishchik, A. G. Tarnavsky, S. B. Zhibinov, S. S. Chesnokov, "Information technologies and problems of the development of the Computer Simulation Center in Internet" (in Russian), Information Technologies, Vol. 8, pp. 68-73, 2009.

[2] G. A. Tarnavsky, E. V. Vorozhtsov, "Computer simulation center in Internet”, Cornell University, New York, 2011. http://arxiv.org/abs/1101.0664.

[3] G. A. Tarnavsky, "Cloud computing: content, infrastructure, and technologies of the arrangement of information flows of the Computer Simulation Center SciShop.ru" (in Russian), Investigated in Russia, Vol. 13, No. 001, pp. $1-29, \quad 2010$ http://zhurnal.ape.relarn.ru/articles/2010/001.pdf.

[4] G. A. Tarnavsky, A. G. Tarnavsky, K. V. Gilev, "Informationcomputation Internet Center "Aeromechanics". The first line: program complex "Shock"” (in Russian), Numerical Methods and Programming, Vol. 6, No. 1, pp. 27-48, 2005.

[5] G. A. Tarnavsky, "Shock-wave modes of flow at the inlet to the diffuser of a hypersonic scramjet engine: The effect of flight altitude and velocity", High Temperature, Vol. 43, No. 1, pp. 58-72, 2005.

[6] G. A. Tarnavsky, A. V. Aliev, A. G. Tarnavsky, "Computer simulation in aeromechanics: program complex "Flow-5"” (in Russian), Aerospace Technology, Vol. 4, pp. 27-38, 2007.

[7] G. A. Tarnavsky, A. V. Aliev, "Peculiarities of the high-speed flight aerodynamics: computer modelling of hypersonic flow around the object forebody" (in Russian), Numerical Methods and Programming, Vol. 9, No. 2, pp. 371-394, 2008.

[8] A. V. Aliev, G. A. Tarnavsky, "Hierarchical SPH-method for mathematical modelling in gravitational gas dynamics" (in Russian), Siberian Electronic Mathematical Proc.,Vol. 4, pp. 376-434, 2007.

[9] G. A. Tarnavsky, V. S. Anishchik, "Toolbox NanoMod for computer support of the design of nanostructured semiconductor materials" (in Russian), Numerical Methods and Programming, Vol. 10, No. 2, pp. 34 $50,2009$.

[10] G. A. Tarnavsky, E. V. Vorozhtsov, "Dopant implantation into the silicon substrate with non-planar surface", Energy and Power Engineering, Vol. 2, No. 2, pp. 73-77, 2010.

[11] G. A. Tarnavsky, "Remote computer simulation of shockwave structures in hypersonic gas flows: technology of cloud computations "The workplace as a service"' (in Russian), Numerical Methods and Programming, Vol. 11, No. 2, pp. 1-25, 2010.

[12] S. B. Zhibinov, G. A. Tarnavsky, "Computer Simulation Center in Internet: problems of copyright and intellectual property of the content" (in Russian), Investigated in Russia, Vol. 12, No. 073, pp. 953-967, 2009. http://zhurnal.ape.relarn.ru/articles/2009/073.pdf. 